\title{
Selective Erasers: A Theoretical Framework for Representing Documents Inspired by Quantum Theory
}

\author{
Alvaro F. Huertas-Rosero \\ Department of Computing Science, University of Glasgow \\ alvaro@dcs.gla.ac.uk
}

\begin{abstract}
The problem of representing text documents within an Information Retrieval system is formulated as an analogy to the problem of representing the quantum states of a physical system. Lexical measurements on text are proposed as a way of representing documents which is akin to physical measurements on quantum states. The representation of the text is only known after measurements have been made, and because the process of measuring may destroy parts of the text, the document is characterised through erasure. These so called "Selective Erasers" provide the basis for representation [1]. During my presentation, the mathematical foundations of such a quantum representation of text will be presented and discussed in the context of indexing and retrieval within a "quantum like" Information Retrieval system. The presentation will then outline the various directions for future work using Selective Erasers.
\end{abstract}

Information Retrieval, Quantum Theory

\section{Acknowledgements:}

I would like to thank my supervisors C. J. van Rijsbergen and Leif Azzopardi for their valuable and fruitful advice. Also, I would like to thank the European Commission for supporting my PhD studies through the K-Space Project (FP6-027026), and the Department of Computing Science of the University of Glasgow for the travel support through the Robert's fund.

\section{References}

[1] Huertas-Rosero, A., Azzopardi, L., van Rijsbergen, C.: "Characterising through erasing: A theoretical framework for representing documents inspired by quantum theory". In: Quantum Interaction 2008, Oxford, U. K., College Publications (2008)

[2] van Rijsbergen, C. J "The Geometry of Information Retrieval" Cambridge University Press, 2004 